\title{
Dilemmata bei der Offenlegung genetischer Daten - Aktuelle Entwicklungen
}

\section{Jean Martin}

Das Recht auf Wissen bzw. unter Umständen das Recht auf Nichtwissen ist eines der grossen Themen der medizinischen Ethik. Seit etwa einer Generation wird grosses Augenmerk darauf gerichtet, dass der Patient das Recht hat, von seinem Arzt alle Informationen zu bekommen, die ihn betreffen - einschliesslich der Patientenakte. Dieser Anspruch hat ganz neue Aspekte bekommen, seit zu immer mehr Gelegenheiten genetische Erkenntnisse gewonnen werden - sei es bei einer In-vitro-Fertilisation (Präimplantationsdiagnostik; derzeit Gegenstand der politischen Debatte in der Schweiz), darauf in der Schwangerschaft, bei der Geburt oder später im Leben.

Ein häufig angeführtes Beispiel ist Chorea Huntington, eine Erbkrankheit mit dominantem Erbgang, die im Erwachsenenalter zu Demenz und Tod führt und für die es keine Behandlung gibt. Die vieldiskutierte Frage in diesem Zusammenhang betrifft die Kinder von betroffenen Menschen: Es gibt ja den Test, der zeigt, ob sie gesund sind oder ein genetisches Merkmal tragen, das für sie einen frühen Tod unter sehr belastenden Bedingungen bedeutet. Natürlich gibt es ein Recht darauf, Bescheid zu wissen oder unwissend zu bleiben, doch andererseits betrifft die Entscheidung auch einen anderen Menschen: das mögliche, ungeborene Kind. Hier wird auch deutlich, wie heikel die Situation für einen jungen Erwachsenen ist, der sich aufgrund einer positiven Familienanamnese testen lässt und herausfindet, dass er Träger des Gendefekts ist, während seine Eltern, die sich nicht haben testen lassen, (noch) nicht krank sind.

Das hier vorgestellte Werk beleuchtet vor dem Hintergrund der Entwicklung der technischen Möglichkeiten sowie der bisherigen Erfahrungen die Fragen, die sich im Zusammenhang mit der Offenlegung genetischer Informationen stellen. «Es haben sich neue Probleme aufgetan, die vor 10 Jahren noch nicht ersichtlich waren, Probleme, die sich vielleicht erst offenbaren, wenn man den Standpunkt des Patienten annimmt und versucht zu ergründen, wie man Dilemmata <empfindet, wenn man sie mit ihren konkreten, lokalen Gegebenheiten und Erfahrungen erlebt» (S. 2). Die Herausgeber sind zwei Professoren aus dem Umfeld der Universität Basel, die 25 Autoren der einzelnen Beiträge kommen aus der Genetik, anderen medizinischen Fächern, Ethik/Philosophie, Rechtswissenschaft und Soziologie. Hervorzuheben sind auch ihre Standorte: Im Gegensatz zu einem grossen Teil der bioethischen Literatur sind hier nur zwei der Autoren in Nordamerika ansässig, die anderen sind vorwiegend auf die Schweiz, Deutschland und Grossbritannien verteilt. Angesichts der deutlichen Nuancen, die beim Thema Bioethik die beiden Seiten des Atlantiks unter-
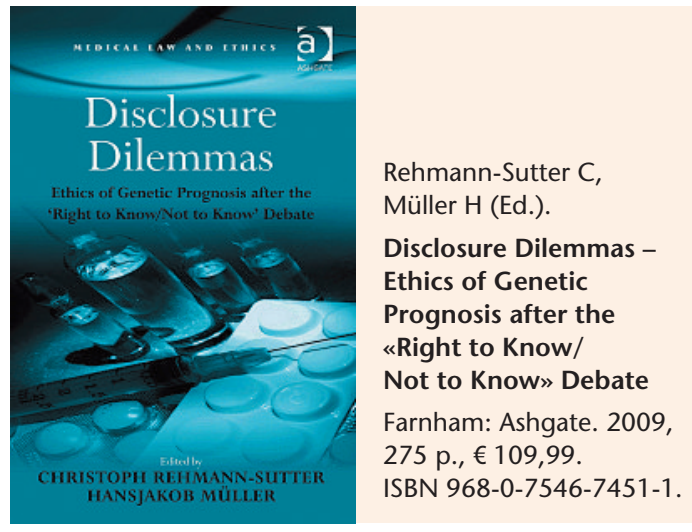

scheiden, wäre es wünschenswert, dass das Buch insbesondere in den USA auf Aufmerksamkeit stösst.

Am Anfang des Buchs ein Zitat aus Schillers Gedicht Kassandra: «Warum gabst du mir zu sehen, was ich doch nicht wenden kann?» Eine berechtigte Frage, die die Gentests aufwerfen!

«Disclosure Dilemmas» ist in drei Abschnitte mit insgesamt 18 Kapiteln gegliedert: Hintergründe (insbesondere zur genetischen Beratung); Fälle/Situationen und Fragen; Verantwortlichkeiten. Die verschiedenen Dimensionen der Verantwortung, die jeder Beteiligte trägt, werden bis ins Detail untersucht. «Wie weit man auch von Vater, Mutter, Bruder, Schwester, Sohn oder Tochter entfernt lebt, emotional sowie geographisch. Selbst wenn die immer häufiger auftretende Patchwork-Familie bedeutet, dass man vielleicht nicht länger in Kontakt zum biologischen Vater steht. Die Entscheidung, einen Gentest durchzuführen, rückt die betroffene Person unweigerlich wieder in eine direkte Verwandtschaftsbeziehung mit ihnen, ob diese Beziehung die Sozialisierung dieser Person geprägt hat oder nicht» (Porz, S. 61).

Es würde natürlich den Rahmen sprengen, hier den über 250 Seiten langen, dichten Text zusammenfassen zu wollen. Im Folgenden sollen einige Anmerkungen gemacht werden.

Dazu, dass vor 20-30 Jahren die übergeordnete Maxime in der genetischen Beratung die Nichtdirektivität war (eine Maxime, an deren Stelle ein breiter gefasster Ansatz treten soll; siehe Anmerkungen zum Konzept der Agency weiter unten): «Die demonstrative Vermeidung von Direktivität gewährleistet sogar moralische Legitimität und wendet den Vorwurf ab, Genetik sei eine Kollektiv- und Zwangsmassnahme auf der Ebene der Bevölkerung bzw. des 〈Gen-Pools〉. Jedoch darf die unter diesen Umständen gewährte Freiheit von Direktivität nur eine eingeschränkte Freiheit sein, gerade gross genug, um dem Rechtfertigungsbedarf 
der biomedizinischen «Verfassung〉 der Gesellschaft zu dienen» (Rehmann, p. 231). Man denkt an die von Michel Foucault beschriebene Biomacht.

Die Juristen unter den Autoren beschreiben, wie die rechtlichen Rahmenbedingungen je nach Land unterschiedliche Modelle der Verantwortlichkeit in der genetischen Beratung hervorbringen. Das ist insofern von Bedeutung, als «dass die juristische Haftung selbst in Einzelfällen nicht einfach mit guten moralischen Argumenten aus dem Weg geräumt werden kann» (S. 129). Langewitz und Cerny schreiben in ihren Kapiteln über das Überbringen schlechter Nachrichten, der Erstgenannte auf der Grundlage seiner Erfahrungen in der Onkologie. Bezüglich der Verantwortlichkeiten, die Getestete wie Fachpersonal übernehmen müssen, zitiert Th. Cerny den französischen Philosophen Jacques Derrida: «Die Verantwortung beginnt genau dort, wo die Sicherheit aufhört.» Mehrere Autoren unterstreichen, dass es (natürlich) keine Patentrezepte gibt, die auf jede Situation passen, und dass Lösungen nicht allein auf theoretischem Wege gefunden werden können.

Kapitel 14 ist ein Beitrag dänischer Autorinnen über die Erfahrungen in ihrem Land mit den Entscheidungen von Frauen/Paaren zu pränatalen Untersuchungen in Risikoschwangerschaften. In mehreren Beiträgen wird betont, dass die Genetik heute weit über die relativ seltenen monogenetischen Krankheiten hinausgeht und den Blick auch auf viele multigenetisch bedingte Krankheiten richtet. Und dass es auch wichtig ist, die neuesten Ergebnisse der Epigenetik zu berücksichtigen.

In seinem Beitrag mit dem Titel «Allowing Agency» präsentiert Rehmann ein Modell für die Kommunikation über genetische Daten, das darauf ausgerichtet ist, die «Agency» zu stärken, die Fähigkeit des Einzelnen, verantwortungsvoll zu entscheiden und zu handeln: «Agency ist ein Begriff, der, wenn er vorsichtig analysiert wird, alle sechs Bestandteile des Modells abdeckt. Die Person muss als Agierende gehört und gewürdigt werden, dass heisst als Subjekt von Evaluation, Verantwortung und des moralischen Handelns. Agency bedeutet, dass die Möglichkeit gegeben ist, Verantwortung zu tragen und zu reagieren, dass man genug Raum hat, unter jeglichem Druck zu bestehen, und frei und fähig ist zu handeln. Das Verständnis dafür, was in der jeweiligen Situation relevant ist und was ernsthaft in Betracht gezogen werden sollte, ist eine Ressource, um dieses Potential ausüben zu können. Agency bedeutet auch, dass der Agierende unabhängig in dem Sinne ist, dass er seine eigenen praktikablen Antworten findet. Jemandem wird agency zugesprochen, wenn er die Praxis angesichts der Werte und langfristiger Pläne für das Leben evaluieren kann mit Verständnis für ein «gutes und erfülltes Leben»» (S. 256).

Im Schlusswort fassen Rehmann und Müller die aufgeworfenen Fragen zusammen, die beantwortet werden müssen, auf der Basis der gewonnenen Erfahrung und gegebenenfalls auch der Fehler, die gemacht wurden ... Die Patienten-Einverständniserklärung hat uns (zu sehr) glauben lassen, wir hätten eine Patentlösung gefunden - diese Position müssen wir überdenken: «Die informierte Zustimmung zu den Tests kann keine Antwort auf die ethische Frage sein, ob diese Tests gerechtfertigt sind oder nicht. Jedoch wäre das Testen ohne informierte Zustimmung hochgradig problematisch und würde die Grundrechte der Getesteten verletzen» (S. 264).

Und: «Aber welches Mass an Offenlegung genetischer Risiken ist für die Menschen gut? Wie hoch ist der Preis dieses Wissens in Bezug auf Wohlergehen und Zufriedenheit? Wie hoch ist der Preis für die Autonomie? Mit anderen Worten: Welchen Wert messen wir der Freiheit bei, auch den irrationalen Aspekten unseres Lebens Gewicht zu geben (zum Beispiel Vergnügen, die gesundheitsschädigend sind)? (...) In was für einer Gesellschaft werden wir leben -, wenn pränatale (bzw. präimplantatorische) Gentests nicht mehr die Ausnahme sind, sondern die Regel?» (S. 266). Hier ist auch an die Vorbehalte zu erinnern, die insbesondere Didier Sicard, der ehemalige Vorsitzende des französischen Ethikrates, und mehrere seiner Kollegen geäussert haben, als es um die (tatsächliche oder geplante) steigende Verbreitung von systematischen Screening-Untersuchungen ging, die ihrer Ansicht nach wahllos vorangetrieben wird [1].

Wie es in den letzten Zeilen des Buches heisst: «In der Zukunft könnten wir diesen Gen-Zentrismus hinter uns lassen. Nicht-genetische Faktoren tragen zum Auftreten von Krankheiten ebenso bei wie genetische. Aber selbst wenn wir eine ganzheitlichere Sicht wählen und daran glauben, dass zwischen sgenetischer Information und «nicht-genetischer> Information kein entscheidender Unterschied besteht, beanspruchen Gentests, wegen ihren praktischen Gegebenheiten doch eine Sonderrolle.» Man denkt an den immer einfacher werdenden Zugang - auch ohne Beratung durch Fachpersonal -, an eine gewisse Unmittelbarkeit der Daten, die als hochvalide betrachtet werden ... obwohl doch, wie nicht genug betont werden kann, selbst wissenschaftliche, reproduzierbare genetische Daten für den Einzelfall immer nur einen relativen Wert besitzen; man darf sich nicht blind auf sie stützen, sondern muss sie interpretieren, unterstreicht H. Müller.

Es steht ausser Frage, dass die Entscheidung für oder gegen Gentests sowie die Beratung dazu und die Konsequenzen, die man aus den Ergebnissen zieht, weiterhin hochkomplexe Fragen aufwerfen. Zur Entwicklung der Genetik in den letzten vierzig Jahren ist auch auf einen interessanten Artikel von Th. Murray in einer aktuellen Ausgabe des Hastings Center Report [2] zu verweisen, und bezüglich des Rechts auf Nichtwissen auf eine Publikation von R. Andorno [3]. Abschliessend soll die Zielsetzung erwähnt sein, die die Serie «Medical Law and Ethics», in der dieses Werk erschienen ist, verfolgt, nämlich darüber zu diskutieren «in welchem Masse Wissenschaft und/oder Medizin die moralische Agenda bestimmen sollten und welche Rolle in diesen Bereichen dem Gesetz zufällt». Eine bedeutsame und dringliche Zielsetzung. 